\title{
Discussion on the Large-scale Collaborative Development and Evolution of Network-based Trusted Software
}

\author{
Wei Yang \\ Shenyang Urban Construction University \\ Shenyang City University
}

\begin{abstract}
Along with the informatization of the global information resources, the Internet network application is more and more extensive. Software has been in every field; the demand for software development is increasingly greater; the software development market is mainly manifested in the modern engineering. Meanwhile, the progress of the times, software development platform, development environment, and development language change accordingly. Different software has been developed on the Internet according to the different environment. A combination of the developed software with the practical work for improving the software productivity and quality is an important issue necessary to think about in the software development field under the Internet age now. The reliable degree of software is also focused by more people. Development and analysis are the foundations of trusted software.
\end{abstract}

Keywords- Trusted Software; Group Collaboration; Resource Sharing; Credible Analysis; Software Evolution

\section{Introduction}

In software development, edition and application, the operating system is the essential, the most fundamental, and the most important system software in all software layers. This is because it is enabled to carry out unified control, scheduling and management on all the hardware and

\author{
Liming Geng \\ Shenyang Urban Construction University \\ Shenyang City University
}

software and data resources. Its main characteristics include concurrency, sharing, and asynchrony. In the current software development, the desired effect is not played if the security and reliability of the software cannot meet the requirements. The "trusted" in the trusted software means that the behaviors and results of an entity are always predictable once a given target is available. If the software service is always in line with the expectation of the users, this type of software is always trusted even if there are some special cases to occur in the running process. The special cases include hardware environment malfunction, low-level software errors, effect from other software, intentional attacks, and unintentional operational errors. What kind of software is trusted? Trusted software is usually supposed to feature easy availability and functions, good reliability, high security (confidentiality, integrity), short response time (from input to output, and low maintenance cost (monitoring, evolution).

\section{Background}

The Internet has been an important information infrastructure now to push the economic development and social progress. Software is the core, but also not always reliable. This is the common "software reliability" problem. The reliability of the traditional software was mainly the impact from the design and construction problems and human errors on the software quality. In the improvement of the traditional software development methods and techniques, the impact from human errors on the software 
quality is more greatly focused; automation and engineering software development technology is mostly applied.

Automation technology is a comprehensive technology and has a very close relationship with cybernetics, information theory, systems engineering, computer technology, electronics, hydraulic pressure technology, and automatic control; control theory and computer technology play the greatest effect on the automation technology.

Software engineering is a discipline emerging in the end of 1960s for solving software crisis, mainly including the software development and maintenance using systematic, standard, and quantified engineering principles and methods. Software development technology includes software development methodologies, software tools and software engineering environment. Software project management includes software metrics, project estimate, schedule control, personnel organization, configuration management, and project planning and so on.

Under the age of the Internet, software technology and application mainly feature development and analysis (evolution) as the basic elements of software development activities and generation. The crystallization of group strength and the reliability-cored evolution play a profound effect on the large-scale collaborative development and evolution of the network-based trusted software. Improving the productivity and quality of trusted software is an issue necessary to highly focus today; the software development breaking the old and traditional technology development is based on the combination of group human errors and trusted evolution technology. The success of open source software is mainly reflected in the following characteristics.

Group collaboration is important. Group collaboration can improve the software development efficiency and quality. Software development technology needs both development and innovation, and its value can be better reflected only if development and innovation are combined together.

Resource sharing is necessary. The sharing is based on the network data and application. In software development environment, the internal resources of a project can be shared, and also the resources outside the project can be introduced, and thus, the software resources are recognized by users in the sharing process, and also the advice from users is adjusted, and then the resources are shared by more users.

The scope of the operating environment is analyzed as follows. The environment of the software is very complicated in the networking running process. Whether the software can adapt to the environment and the due changes, analyze the system status of other software, reduce the errors in the system running, and timely respond the dynamic update and problems is the key to improve its trusted evolution in its running process.

Reliability analysis is as follows. High-quality software can be created only if it is constantly evolved and updated; users can provide the related reference opinions on the software at the network platform; the software is edited and updated within a certain period of time. Therefore, the lifecycle of the software in management, analysis and optimization is the foundation of improving the software reliability.

Group collaboration is a new process of trusted software and changes the understanding of software development. On the basis of the network, group ways are trusted and feasible in software development, and also provide the resource sharing, group cooperation, and credibility analysis of software development environment, so as to implement software innovation, deep collaborative production, adequate software sharing, and the regular update of the software collaborative process. Thus, the large-scale collaborative development and evolution of the network-based trusted software is implemented. 


\section{Reliability-based trusted software model and evolution}

Reliable evidence can be implemented through the data used in the software development; whether the designed software is reliable and feasible can be determined according to the service condition for users, information feedback, and ranking after it is released on the Internet, or according to some measuring instruments in its application process. To determine the reliability of software based on the feedback of users is the most feasible and also the most easily accepted; the designed software will be very popular among the users. Therefore, trusted software is built on the basis of variety of credible and feasible developments.

A software tool needs constant modification, update and edition and then withstands any test. Trusted software evolution refers to a process to make changes for constantly meeting the user needs. Under the network environment, software development, design, and editing codes also change based on the user feedback, and thus, the requirement for the software is higher and higher. Ultimately, the software version is constantly updated; the reliability of the software is improved; the operating model is optimized.

\section{Discussion on the large-scale collaborative development and evolution of trusted software}

The large-scale collaborative development and evolution of trusted software is mainly oriented at the network-based basic software; the formation, collection, sharing, and application reliable evidences at the three stages of software collaborative development, evolution and application are supported; the software trusted evolution is accelerated, so as to improve the development efficiency and application quality of trusted software. The service system structure is centered at reliable evidences and completed through group collaboration, resources sharing, running environment control, and reliability analysis. Software resource sharing is divided into the initial code sharing and the user reference opinion sharing. To share the source codes, the software version is updated; new ideas are excavated. Then, the shared opinions can be used for effective references so as to design effective and practical software. These can effectively accelerate the reliable evolution of trusted software. Software is composed of three phases: development, operation, and application. Reliability analysis can be made from initial data, operating data, and resource reliability. To develop a project, effective analysis and calculating various kinds of data required by the project form the initial data. Then, the availability of the project can be achieved. The software developed after data analysis may fail to work in the running process. The problems occurring in the running process are necessarily taken into account in the project development process and also a related solution is found. Generally, all aspects are considered and emphasis may be different when software is developed. In this case, the primary and secondary function of different data is necessarily analyzed, so as to achieve the main requirements of the development project.

\section{The large-scale collaborative development environment for trusted software}

The development of trusted software is necessarily implemented in different environments based on community model or social network model. To socialize the development environment, software and social environment are flexibly used. The social environment refers to a tool for exchanges and sharing, namely, a network tool such as BBS, communication tool, and email, or an industrial production tool such as project management, development, and application. To share the trusted software, right and credible resources are collected. In the development environment of software, resources sharing can be implemented; the expected effects of users on the software can 
be collected, analyzed and utilized, in order to secure the reliability and availability of the developed software.

\section{The future prospects}

With the constant changes of the information age, the people's demand is varying without a stop; the demanded contents are diversified; all kinds of different things emerge. More and more software tools are developed; the software development converts from the simple to the complex and various and from small size to large size; the development environment changes from the static to the open, dynamic and complicated. The software reliability and security are receiving more and more attention from people; the practicality of the software designed and constructed by people is improving, so as to reduce the people's errors and the invalidation of capital investment, and increase the software reliability, and then implement the large-scale development and evolution of the network-based trusted software.

\section{Conclusion}

In the Internet open environment, software development, analysis and application should be expanded and extended and combine theory with practice, but not be limited to a particular aspect.
The large-scale development and evolution of the network-based trusted software can secure a project to work normally, optimize, share and become reliable. Trusted software is the new technology of information system security, which is a new area of the international information security. It is necessary to adhere to technological innovation, the combination of theory with practice, and a wide application. The field of the Internet is necessarily further expanded in China. Opportunities are necessarily seized for enhancing the development and application of reliable software.

\section{References}

[1] Donghong Liu, Changguo GUO, Huaimin Wang, Tao Wang. Monitoring Enabled Distributed Software Construction Method [J]. Journal of Software, 2011 (11)

[2] Lin Yuan, Huaimin Wang, Dianxi Yin, Xiang Li. Exploration and Analysis on the Behavioral Characteristics of the Developers under the development environment [J]. Journal of Computer, 2010 (10).

[3] Xinxing Luo, Mingxun Zhu, Zhongjun Tang. Trusted Software Requirements Acquisition and the Analysis Were Review and Prospects [J]. Computer Application Research, 2010 (10). 